\title{
A New Approach to Competence-Based Business Partner Profiles for Collaborative Business Process Management
}

\author{
Thomas Schuster and Peter Weiß \\ FZI Forschungszentrum Informatik, Haid-und-Neu-Straße 10-14, \\ 76131 Karlsruhe, Germany \\ \{weiss, schuster\}@fzi.de
}

\begin{abstract}
In this paper a novel approach and thinking to foster the derivation of competence and skill requirements by business process models is presented. Management of intra- and inter-organizational business processes is a significant issue that influences a company's success. A multitude of competence models, standards and frameworks exist. Furthermore, current resource models in business process management solutions are not sophisticated and adequately reflecting the needs of collaborative resource planning and competence management. Our research activities yielded a meta model which tends to overcome existing shortfalls. In this article we present an extensible resource meta-model that allows the modeling of resources including their competences, skills and knowledge. Furthermore we demonstrate the aggregation of competence concepts to business partner profiles in collaborative networks.
\end{abstract}

Keywords: Collaborative Resource Management, Competence, Business Process Management, Ressource Allocation.

\section{Introduction}

The selection of business partners can be identified as key to success and key capability for the realization of collaborative networks in near future [15, 14]. Against this background the paper looks at competence management and related models in the context of business process engineering. In particular our approach fosters the alignment of business process modeling and human resource planning. Previous research has already motivated the debate to link competence theory and business processes [12]. While it is worthwhile to look at competence models and business processes from a single company perspective, the added value from an intercompany perspective shows to be even higher. The remainder of the paper is structured as follows: first the current state of the art and basic concepts are introduced. Consecutively an extensible resource meta-model comprising subsets of meta models (workflow systems, human resources and competence) is presented. Competencebased and resource-based views in the organization are motivated and an example for collaborative competence-modeling is presented to illustrate how the model shall be applied. Finally, we summarize the achievement and provide an outlook on next steps and future research. 


\section{Collaborative Networks and Business Process Management}

In the past, research made a clear distinction of business process perspective and competence-based management views. Whereas, workflow management rather relates to the operational and functional view, competence management is hitherto associated with the HR domain and recruitment processes. Collaborative networks comprises a interdisciplinary research community which has elaborated in the meanwhile a comprehensive knowledge base encompassing theoretical foundations, services, collaboration and innovation environment, and last but not least the analysis of the impact collaborative networks have on daily life and economy [15]. Competence management and a resource-based view on collaborative networks get popular and subject of related research $[12,15]$. The selection of business partners is considered as a key to success for the realization of collaborative networks in near future. Assessment of competences can be seen as integral part of partner selection processes and partnership formation.

"An allocation relates tasks, resource classes, case attributes, and resources to each other" [13, p. 81]. In this context a collaborative resource management formulates and analyses preconditions of allocation policies, which can become highly complex. Therefore a formal approach and machine readable format is required. Resources available in a collaborative network to carry out the workflow typically are classified and decoupled from specific employees. Today resource classification is realized solely based upon two dimensions: role models and organization chart. The latter contains organizational units and hierarchical structure. Role models are in most cases derived from the functional structure of an organization [13, p.77]. Most workflow management systems provide graphical resource classification tooling [13, p.153].

In this paper, we propose a novel approach to classify resources based on business partner profiles (BPP) [15] extended through role profiles. The latter include qualifications and capabilities a resource may require to perform an activity in expected quality and time. BPP represent information required to discover, select, and integrate business partners into emerging or existing value chains $[15,14]$. Job profiles are built according to specific company and work place needs. A job profile can be specified by a set of competences and related descriptors for skills, knowledge and attitude. Descriptors relate to an internal or external skill framework. To specify and store job profiles standards such as $[11,4,5]$ may be used. Resource classes are allocated to workflows through analysis of the characteristics and requirements of an activity in relation to a specific case [13, p.80]. In our approach information stored in job profiles is used in addition to enhance the allocation of resources to specific cases. On one hand it is necessary to extract requirements of business partners' competences (from strategic to individual levels such as performance levels, proficiency levels, qualification and training) from the business processes, on the other hand metrics that allow for the matchmaking or alignment of these requirements with available roles or job profiles of companies are needed. In this way, workflow management can profit from sophisticated software services allowing job profile aggregation, comparison and alignment to specific tasks. Within companies, the recruiting process usually involves at least three interested parties [11]: line management, HR department, and potential applicants (internal or external to the company), all of them need to clearly understand the specification of the job, the company, compensation and, benefits. In 
collaborative networks those functions may be as well broken down into tasks and distributed between network nodes and resources. These tasks can be again aggregated to respective collaborative processes. Facilitating communication between these three functions is a key issue in the effectiveness and success of the recruitment process [11]. A meta model of competences is a prerequisite to allow inter-company interaction and information exchange.

\section{Analysis and Design of Proposed Solution}

In this section we illustrate requirements and the derived conceptual design of the proposed solution. Consequently we outline a meta model for competences. Furthermore we strive to reveal relationships of competence models [16] (in a HR resource view) and resource models (a workflow management view). Supplementary existing standards that have influenced the conceptual model (such as the European eCompetence Framework (e-CF) and HR-XML) are pointed out. Our conceptual model [7] is based on a MOF-compliant meta-model [9] that is built on three parts, the resource meta-model (RMM - containing general aspects of resources such as allocation state, current location etc., nevertheless details of the RMM will not be covered in this paper), the human resource meta-model (HRMM) and the competence meta-model (COMM).

Figure 1 illustrates details of the human resource meta-model (HRMM). HRMM combines common resource meta-models as known in workflow management $[13,12]$ with competence descriptions. The meta-model allows precise definitions of organizational aspects and hierarchies, furthermore it enables an explicit by descriptions of competences. Competences can be modeled independently and may be reused for further specifications of roles and human resources. The specification of competences is covered by the competence meta-model (COMM). As a key part of HRMM the human resource (HumanResource) depicts manpower. In order to represent the

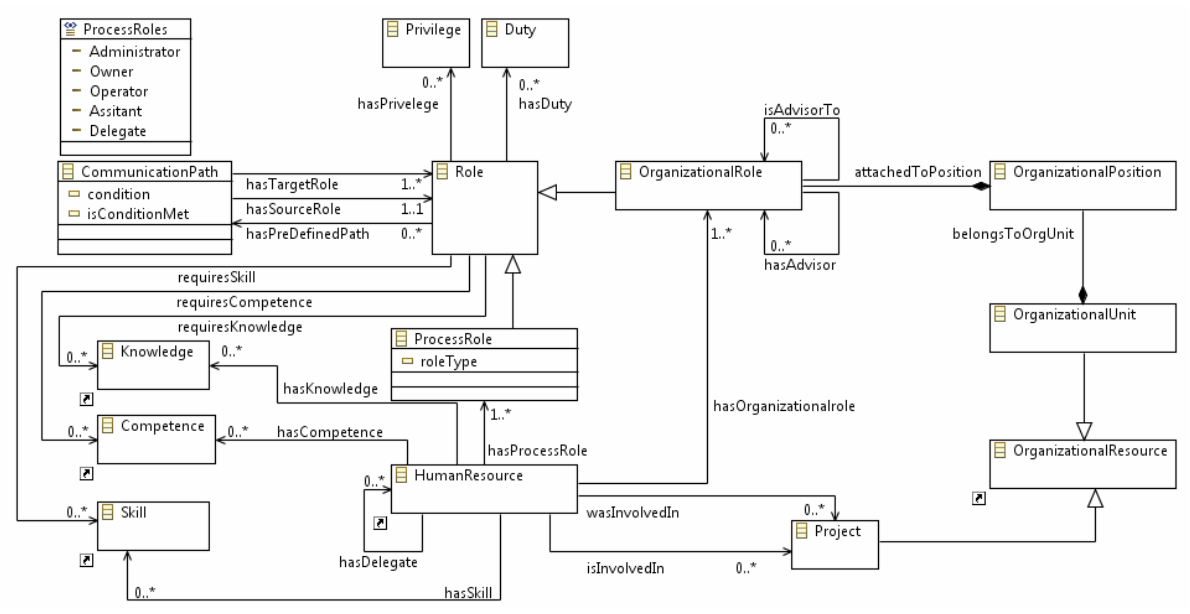

Fig. 1. Human Ressource Meta Model (HRMM ) 
organizational structures a human resource can be associated to an organizational role (OrganizationalRole) [3,12], furthermore in a business process context an association to a process role (ProcessRole) [3] is possible. Organizational hierarchies are reflected by OganizationPosition, OrganizationalUnit and the relations hasAdvisor and isAdvisorTo. The business process-related role (ProcessRole and the accordant enumeration ProcessRoles) is used to describe the relationship between human resources and assigned tasks [10,12]. Organization-related roles can be used to determine appropriate resources for task execution. Both role types share properties of their super class Role. These properties are rights (Privilege), obligations (Duty) and defined communication channels (CommunicationPath), e.g. for reporting or escalation mechanisms [13,6]. The model elements OganizationPosition and OrganizationalUnit substantiate organizational role descriptions of the type OrganizationalResource, referenced directly from the RMM part.

The details of HRMM stated above enclosed organization-, project- and processrelated data, these aspects are covered by most common resource models in workflow management [3]. Furthermore a number of studies state that roles [13,12] and human resources also include specific competences [11,4], however these are not modeled explicitly so far. HRMM integrates competence descriptions and associates them to roles and human resources in order to tackle this concern. These descriptions are represented by the model elements Competence, Skill and Knowledge, that are reused elements specified by the competence meta-model outlined below. Clearly this bridges the gap between business process management and human resource management by a precise and formal connection mechanism. In consequence this mechanism does not only reveal details about the modeled resources and their competences, but also enables decision support for a multitude of questions. Decisions that may be supported by this modeling technique are: (1) matching between resources and roles with sound decisions for the allocation of new or idle roles; (2) allocation of resources to tasks in business processes, not only role based but also competence driven assignments; allowing enhanced scheduling mechanisms compared to role based scheduling. The utilization of competences to assign resources to tasks may restrict or extend the selection of acceptable resources. (3) Identification of core competences, driven by analysis of competences needed to execute the business processes. (4) Identification of additional competences needed to perform business goals, either resulting in recruitment of new resources, engagement of subcontractors or further education of resources. As previously discussed, competence models are mainly used by HR to accompany recruitment processes, thus being completely separated from business process modeling. RMM tackles this issue, since it is designed to integrate both model types thereby exploiting potential synergies. Besides the reuse of competence models in business process modeling, derived requirements from business process models may be included in competence models vice versa, thereby improving correlative HR competence profiles with enhanced accuracy. The competence meta-model (COMM) as depicted in Figure 2 specifies competence [16] concepts and relationships. COMM was influenced by several competence standards, such as HR-XML [4], the e-CF [11] and eCCO [2]. 


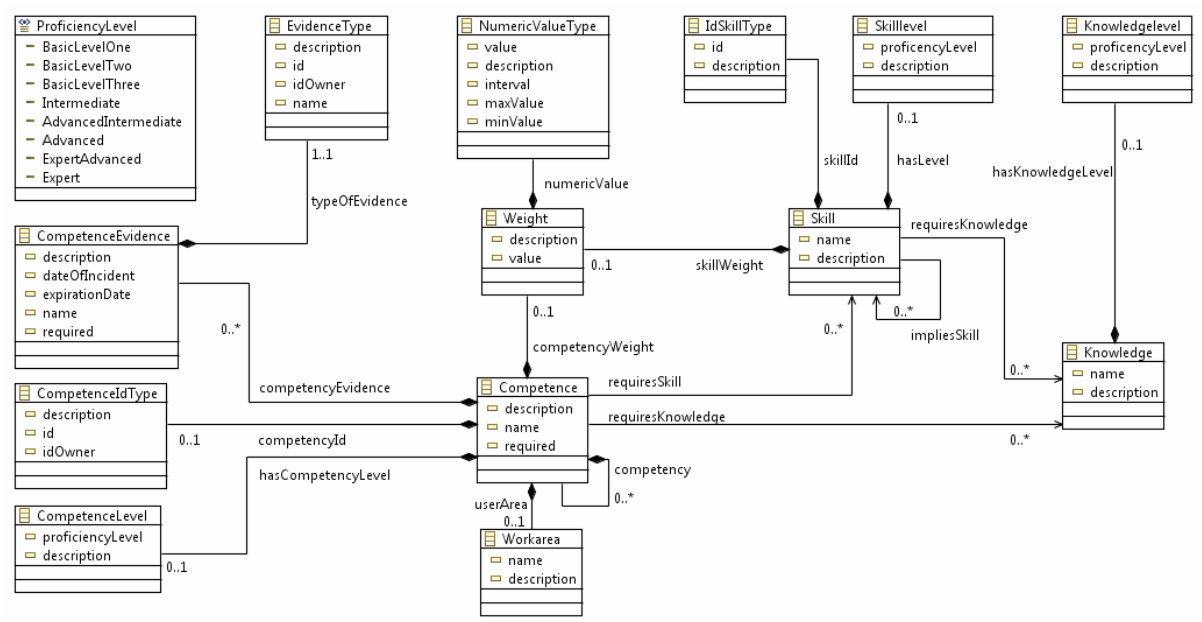

Fig. 2. Competence Meta-Model (COMM)

The basis was derived from HR-XML, since this is not only widespread, but also defines a precise model through the usage of XML schema. Existing HR-XML descriptions may be the input of transformations to create COMM-based models. Additionally the integration of other standards allows a transformation of those descriptions, as we will demonstrate in our use case. The essential elements of COMM are Competence, Skill and Knowledge. According to COMM a competence may imply further competences, require various skills (requiresSkills) and knowledge (requiresknowledge). This relationship between competences, skills and knowledge is an extension to HR-XML according to [2]. In COMM each of these concepts has attached level descriptions that describe the degree of proficiency (proficiencyLevel). Furthermore a competence can be valid in a particular context (Workarea) only, in turn it is invalid in other areas. Additionally competences can be associated with a proof of evidence (CompetenceEvidence). The evidence states dates of achievement, duration of validity and a general description for characterization. Finally, the association of weights to competences and skills is used to measure their importance for an enterprise or a business process. The extensible metamodel design allows to add furthers means for measurement of different aspects in the future (e.g. for scheduling).

\section{Collaborative Competence Modeling}

In order to exemplify the practical relevance of this approach, we present a recruitment process in collaborative scenario. In Figure 3 distinct Conversations between collaborating participants (Consignee; HR department; IT department; Personnel Consultant; Advertising Agency; Career Platform; Job Candidate) of the recruitment process in a partner network are depicted by a BPMN 2.0 Conversation diagram [8]. 


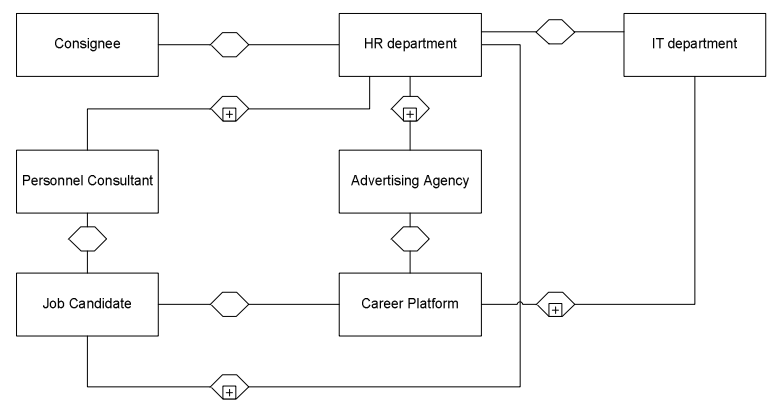

Fig. 3. Conversational Overview Recruitment Process

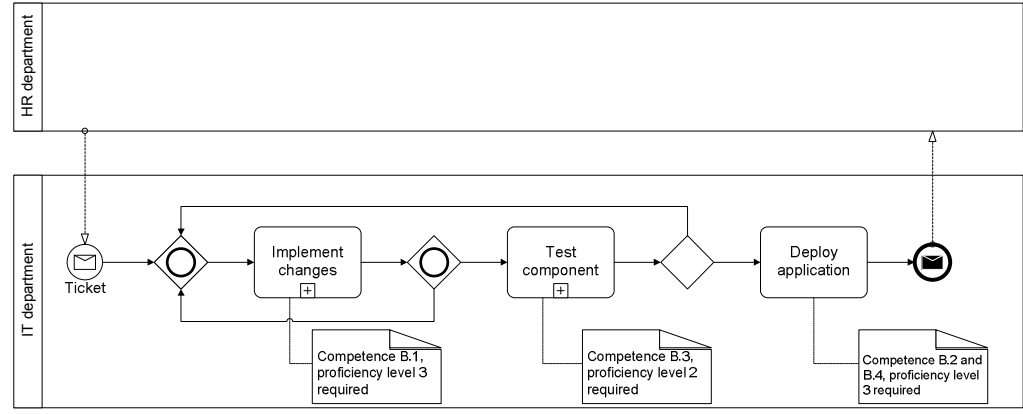

Fig. 4. Example Process - Ticket Processing

At this level competence profiles can be added to the participants. In order to exemplify how these can be aggregated, we will first step into process details by examination of a Collaboration between the HR department and the IT department (Figure 4). In this case the Collaboration is defined as the handling of service tickets, which may lead to changes in a system used by HR to generate adverts in a career platform. The Collaboration (the IT department's view) is quite simple, changes are implemented according to incoming tickets. In a sequential order changes are tested and afterwards made available to the user by redeployment of the application. Development will be continued, if testing fails or further changes need to be implemented, otherwise the ticket processing is completed by sending a message to the issuing partner (HR department). The Collaboration in Figure 4 is extended by the annotation of competences and proficiency levels, these are competence descriptions given by e-CF. The tasks may be assigned to employees from the IT department (specific roles are not defined in this example), the assignment is constrained by the annotated competences. Thus only employees with according competences may execute the tasks.

In Figure 5 a) an organizational description is given as HRMM model with annotated competences, in Figure $5 \mathrm{~b}$ ) the competence Testing is exemplified as COMM diagram. The skills and knowledge in Figure $5 \mathrm{~b}$ ) are defined additionally to e-CF. In the COMM diagram the proficiency level is mapped to an attribute of the competences. Thus HR competence definitions may be reused in business process 


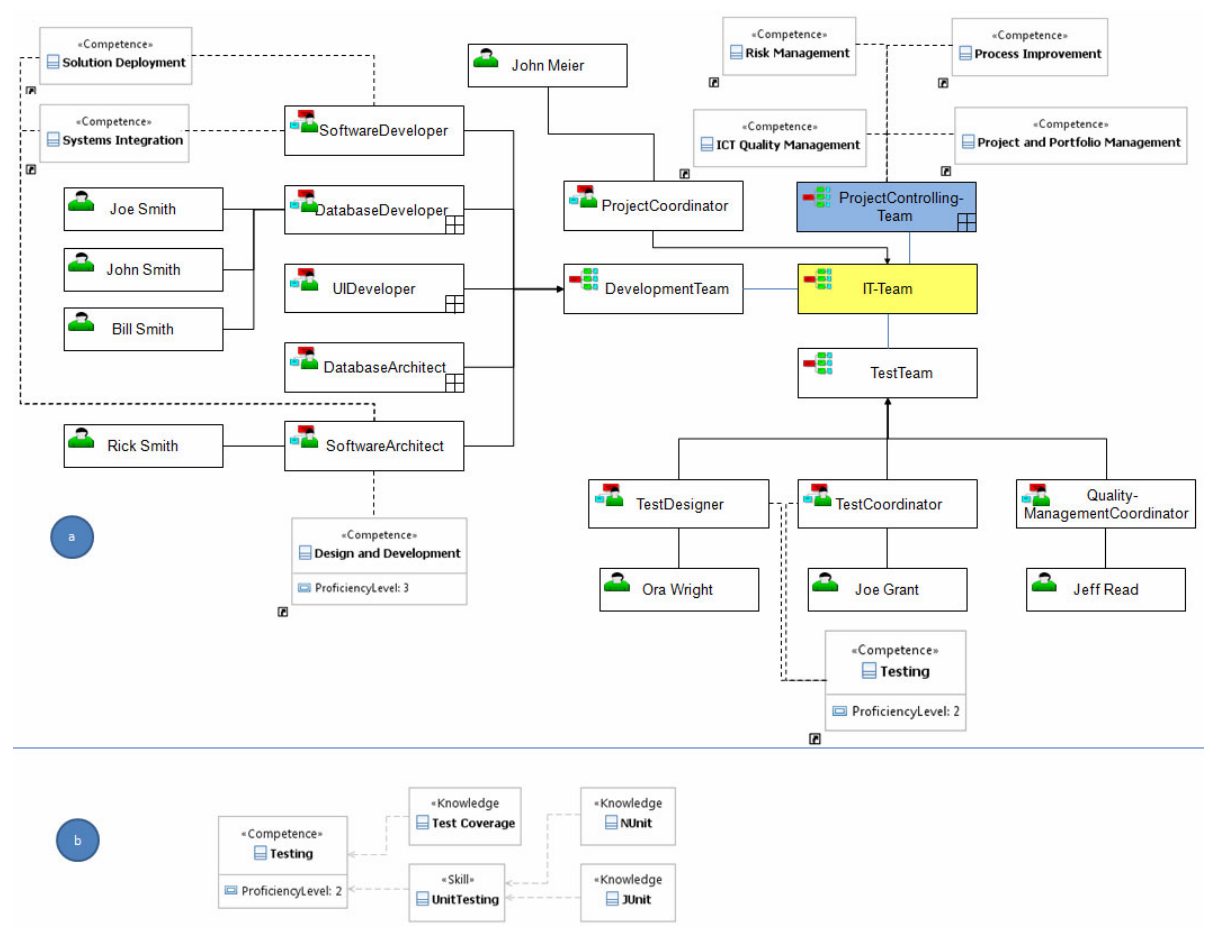

Fig. 5. a) IT-Team as HRMM Model b) Competence B.3 as COMM Model

models, in particular this information may now be used for scheduling. In order to generate partner profiles the competences from the Collaboration level may be aggregated on the Conversation level. If competences do occur repeatedly in Collaborations, the highest proficiency level has to be integrated in the partner profile.

\section{Summary and Outlook}

In collaborative networks data exchange between several IS and the challenge to map available data and information concerning job roles and profiles comes to the fore. Standards to ensure exchange competence requirements and definitions between network companies are needed. On basis of the presented meta-model interoperable resource profiles can be described. Effects of this analysis can be plentiful. We outlined aspects of resource and business process modeling associations between both modeling disciplines. The presented resource meta-model demonstrates the potential of the combination and extension of known concepts in resource modeling, competence modeling and business process management. Competence and resource models of human resource departments can be re-used for the modeling of business process relevant resources. Furthermore existing competence models can be adapted to the actual needs of the business processes. These adapted competence models can in turn be used to improve recruitment of new employees or to set up measures for further education of existing personnel. To extend concepts outlined in this paper, we plan to 
develop mechanisms for competence gap analysis and automatic aggregation of competence profiles. In addition, we plan to enhance our meta-model by industry-sectorspecific features. Finally we strive to generate resource models automatically by the usage of transformations to combine information provided by directory services with existing competence models.

\section{References}

1. Camarinha-Matos, L.M., Paraskakis, I., Afsarmanesh, H. (eds.) Leveraging Knowledge for Innovation in Collaborative Networks, Proceedings of 10th IFIP WG 5.5 Working Conference on Virtual Enterprises, PRO-VE 2009, Thessaloniki, Greece, October 7-9, p. 307. Springer, Heidelberg (2009)

2. eCCO: A European e-Skills Meta-Framework. Draft Proposal. AICA, Federcomin, Fondazione Politecnico di Milano (2005)

3. Hollingsworth, D.: The Workflow Reference Model: 10 Years On, Workflow Management Coalition (WfMC) (2005)

4. HR-XML Consortium: Competencies (2007/2009), http: / /www . hr-xml . org/

5. IEEE Learning Technology Standards Committee: RCD: Draft standard for Reusable Competency Definitions (RCD) (March 2006)

6. OASIS: OASIS WS-BPEL Extension for People (BPEL4People) Technical Committee; Version 1.0 (2007)

7. Oberweis, A., Schuster, T.: A meta-model based approach to the description of resources and skills. Appears in 16th Americas Conference on Information Systems, AMCIS 2010 Proceedings (2010)

8. Object Management Group: Business Process Model and Notation (BPMN) Specification Version 2.0 Beta 1 (2009)

9. Object Management Group: Meta Object Facility (MOF) Core Specification OMG Available Specification Version 2.0 (2006)

10. Russell, N., van der Aalst, W.M.: Work Distribution and Resource Management in BPEL4People: Capabilities and Opportunities. In: Bellahsène, Z., Léonard, M. (eds.) CAiSE 2008. LNCS, vol. 5074, pp. 94-108. Springer, Heidelberg (2008)

11. The European e-Competence Framework, CEN Workshop Agreement (CWA 15893 (part 1 \& 2), CEN (November 2008), http://www.ecompetences.eu/ (visited 17.03.2010)

12. van der Aalst, W.M.P., Kumar, A., Verbeek, H.M.W.: Organizational modeling in UML and XML in the context of workflow systems. In: Proceedings of the 2003 ACM symposium on Applied computing, Melbourne, Florida, pp. S603-S608 (2003)

13. Van der Aalst, W., Van Hee, K.: Workflow Management: Models, Methods, and Systems. MIT Press, Cambridge (2004)

14. Weiß, P., Klink, S.: Collaborative services to maintain electronic business relationsships Establishing the Foundation of Collaborative Networks. In: Establishing The Foundation of Collaborative Networks, Eighth IFIP Working Conference on Virtual Enterprises, Guimarães, Portugal, September 10-12, vol. 243, pp. 435-442. Springer, Heidelberg (2007)

15. Weiß, P., Klink, S.: Social Impact of Collaborative Services to Maintain Electronic Business Relationships. In: Cruz-Cunha, Oliveira, Tavares \& Ferreira (eds.) Handbook of Research on Social Dimensions of Semantic Technologies and Web Services, XXXII, vol. 1, pp. 643-674. IGI Global (2009)

16. Winterton, J., Delamare- Le Deist, F., Stringfellow, E.: Typology of knowledge, skills and competences: clarification of the concept and prototype. In: Cedefop 2005 (2005) 